\title{
OBSERVATIONS ON HORIZONTAL SYMMETRIES AND FLAVOR CHANGING NEUTRAL CURRENTS
}

\author{
D.R.T. JONES, G.L. KANE and J.P. LEVEILLE \\ Randall Laboratory of Physics, University of Michigan, Ann Arbor, MI 48109, USA
}

Received 3 November 1981

\begin{abstract}
We present a detailed study of the possibility of having a horizontal gauge symmetry, in addition to $\mathrm{SU}(3)_{c} \times \mathrm{SU}(2)_{\mathrm{L}} \times \mathrm{U}(1)$. Grand unification is not used as a constraint. We concentrate on the horizontal group $\mathrm{SU}(3)_{\mathbf{H}}$. There are two main results. (i) The horizontal symmetry might break on two mass scales, one heavy and the other of order $m_{\mathrm{Z}}$ or a few $m_{\mathrm{Z}}$. The $\mathrm{K}_{\mathrm{L}}-\mathrm{K}_{\mathrm{S}}$ mass difference and other rare kaon and muon transitions are protected by a residual symmetry, but other flavor changing processes not involving the lightest fermion of each charge can be orders of magnitude larger, e.g. $\mathrm{b} \rightarrow \mathrm{s} \ell^{+} \ell^{-}, \tau \rightarrow \mu \ell^{+} \ell^{-}, \mathrm{e}^{+} \mathrm{e}^{-} \rightarrow \mathrm{b} \overline{\mathrm{s}}, \tau \mu$, tc (while $\mathrm{b} \rightarrow \mathrm{d} \ell^{+} \ell^{-}, \tau \rightarrow \mathrm{e} \ell^{+} \ell^{-}$ and some others are forbidden). Some sectors should have relatively large flavor changing neutral currents. (ii) There is not enough new physics in the approach to explain fermion masses, but the structure of the model suggests that the lighter masses and the KM quark mixing angles might be generated radiatively. Unfortunately, careful analysis shows that the same symmetry that protects the $\mathrm{K}_{\mathrm{L}}-\mathrm{K}_{\mathrm{S}}$ mass difference requires that the Cabibbo angle be identically zero to all orders. This suggests that it may be extremely difficult to make a realistic fundamental or effective SU(3) horizontal symmetry. While the model fails to generate the CKM angles, it is consistent with all low energy data and suggests a number of interesting reactions that might occur.
\end{abstract}

\section{Introduction}

The reason for the existence of flavors is presently a great mystery. While there has been considerable work, no interesting insights have been gained. For instance, people have tried looking for larger groups $[1,2]$ which contain $\mathrm{SU}(5)$ or $\mathrm{SU}(3) \times$ $\mathrm{SU}(2) \times \mathrm{U}(1)$, discrete symmetries [3], horizontal gauge groups $[4,6]$, and other alternatives. There have been encouraging efforts [5] to see flavor as a necessary consequence of a constituent structure for quarks and leptons, but so far no explanation for the number of flavors has come out of that approach.

In this paper we take a conservative approach and study in some detail whether a reasonable description of the low energy domain can be given in a model with a gauge group $\mathrm{SU}(3)_{\mathrm{H}} \times \mathrm{SU}(3)_{\mathrm{c}} \times \mathrm{SU}(2)_{\mathrm{L}} \times \mathrm{U}(1)$. Since there are three known families, a horizontal $\mathrm{SU}(3)_{\mathrm{H}}$ with the three states of a given charge in the fundamental representation is an obvious thing to study. While it has been considered previously $[6,7]$ it has not yet been extensively examined. Such a symmetry could be fundamental, or it could arise as an effective low energy symmetry in a more sophisticated theory. Stated very simply, our conclusions are on the one hand that it is unlikely that a horizontal SU(3) could be a workable symmetry, while on the 
other hand some features of the model suggest interesting mechanisms that might occur in nature, even if the particular model considered is not successful - particularly the occurrence of a light mass scale for horizontal bosons (and consequently some large flavor changing neutral currents ${ }^{\star}$ ).

All of our mass generation is done with a standard Higgs mechanism. Simplicity, and the possibility of dynamically generating the needed Higgs multiplets, are the two criteria used to choose Higgs representations. It is necessary to have a horizontal octet and singlet of $\mathrm{SU}(2)_{\mathbf{L}}$ doublets, which simultaneously give mass to the fermions and give mass of order $m_{\mathrm{Z}}$ to all gauge bosons. In addition it is necessary to have a horizontal sextet of $\mathrm{SU}(2)_{\mathrm{L}}$ singlets which simultaneously can give a large mass to some horizontal gauge bosons and to the right-handed neutrinos (which must be present to cancel anomalies). Using some assumptions, we find a minimum of the Higgs potential and the associated gauge bosons mass matrix.

If only an octet, or only a sextet, were used to generate horizontal gauge boson masses, it is known [8] that $\mathrm{SU}(3)_{\mathbf{H}}$ breaks leaving an unbroken $\mathrm{SU}(2)_{\mathbf{H}}$ or $\mathrm{SO}(3)_{\mathbf{H}}$, i.e. three massless horizontal gauge bosons. When both are present, the Higgs potential depends on their relative orientation (the octet orientation is essentially fixed by the fermion masses). For a wide range of parameters, the three massless gauge bosons acquire mass of order a few $m_{\mathrm{Z}}$. The $\mathrm{SU}(2)_{\mathrm{H}}$ is broken, but a global U(1) survives, and two of the three have equal mass while the third is split off. Which two have equal mass is determined by the original octet and singlet, which basically must give a large mass to the heaviest fermion generation and little mass to the others. Fortunately, then the two bosons with equal and light mass give precisely cancelling ${ }^{\star \star}$ contributions to the $K_{L}-K_{S}$ mass difference and to kaon and muon rare decays.

At this stage the entire approach looks extremely interesting - a possible horizontal symmetry, with lots of testable predictions (see below), subtle mechanisms to protect certain rare decays, no disagreements with experiment. Unfortunately, we would require more of a serious horizontal symmetry. While accounting for the origin of masses is beyond the scope of this approach, the quark mixing angles ought to be expected as output. Having chosen a diagonal input mass matrix, we should expect one-loop off diagonal mass terms $d \leftrightarrow s$, etc., which would then generate the Cabbibo-Kobayashi-Maskawa (CKM) angles. But the same symmetry which protects the $K_{L}-K_{S}$ mass difference guarantees that these are zero whenever a single $\mathrm{d}$ (or $\mathrm{u}$ ) quark is involved. Thus, to the extent that the mixing angles are not generated by another mechanism, the model is a failure.

Since the model is not entirely successful one cannot know what aspects to take seriously for experimental suggestions or theoretical structure. However, in view

* Recent studies of flavor changing neutral currents (which we will denote by FCNC) are in refs. [9, 10].

$\star \star$ This mechanism also occurs in the SU(2) model of ref. [6], and has been considered in a general way in ref. [10]. With the $\mathrm{SU}(3)_{\mathrm{H}}$ model we have the possibility of cancellations occurring in interactions of some flavors but not for others. 
of the total lack of understanding of the generation problem we think that the theoretical mechanisms may be worth investigating, and that the experimental suggestions are encouraging.

Without some positive numerical input of some sort, either a flavor changing neutral current or a neutrino mass or a violation of $e / \mu / \tau$ universality, we cannot give firm predictions for the large flavor changing neutral currents. Consequently, we will give an estimate for the size they might be, taking into account the couplings, gauge boson mixing, and assuming the light horizontal gauge bosons are a few times heavier than the $Z^{0}$.

Which processes occur is clear in the model, though of course whatever mechanism introduces quark mixing angles will modify the situation. It is plausible, however, that these modifications will be small (of order the Cabibbo angle) so there is some value in searching for the modes predicted by this model. There are decays such as $\mathrm{b} \rightarrow \mathrm{se} \mathrm{e}^{+}, \mathrm{s} \mu^{+} \mu^{-}, \mathrm{s} \tau^{+} \tau^{-}, \mathrm{ss} \overline{\mathrm{s}} ; \tau \rightarrow \mu \mathrm{e}^{+} \mathrm{e}^{-}, \mu \mu^{+} \mu^{-} ; \mathrm{t} \rightarrow \mathrm{ce}^{+} \mathrm{e}^{-}, \mathrm{c} \mu^{+} \mu^{-}, \mathrm{c} \tau^{+} \tau^{-}$and the reversed processes such as $\mathrm{e}^{+} \mathrm{e}^{-} \rightarrow \mu \tau, \mathrm{e}^{+} \mathrm{e}^{-} \rightarrow \mathrm{b} \overline{\mathrm{s}}, \mathrm{e}^{+} \mathrm{e}^{-} \rightarrow c \bar{t}$. Note $\tau \rightarrow$ eee, and $\mathrm{b} \rightarrow \mathrm{de}^{+} \mathrm{e}^{-}$. Neutral currents for e, $\mu, \tau$ will not be the same. Since the horizontal theory is vector-like, the horizontal gauge bosons give only vector currents. Readers mainly interested in phenomenological questions could jump to the last section of the paper.

A useful review of the implications of several approaches to horizontal symmetries for rare muon-number-violating $\mathbf{K}$ decays is given by Herczog in ref. [14].

As far as we are aware, there have been two other attempts to look in some detail at an $\mathrm{SU}(3)_{\mathbf{H}}$ symmetry. Chikashige, Gelmini, Peccei, and Roncadelli [7] began with largely the same motivation as ours, but quickly emphasized a different direction - they assigned the left-handed $q=\frac{2}{3}$ quarks and the right-handed $q=-\frac{1}{3}$ quarks to $\mathrm{SU}(3)_{\mathrm{H}}$ triplets, but the right-handed $q=\frac{2}{3}$ and left-handed $q=-\frac{1}{3}$ quarks to $\mathrm{SU}(3)_{\mathrm{H}}$ antitriplets. Consequently they are not studying a direct product symmetry. They emphasize the fermion (including $\nu_{R}$ ) masses and lepton number violation rather than the flavor changing interaction structure.

Yanagida, in a series of papers [6], has given nice discussions of several aspects of horizontal symmetries. He has looked in some detail at $C P$ violation, neutrino masses, and fermion masses in general. Although we agree with him on the form of the fermion mass matrix required by octet Higgs fields, we have dealt with the problem in a different way; we do not attempt to explain fermion masses, so we construct the fermion mass matrix to agree with experiment. He did not discuss the major features of our work, first that the horizontal gauge bosons could come on two mass scales, giving flavor changing neutral currents of quite different sizes, and second that approaches like ours fail to generate the Cabibbo-KobayashiMaskawa (CKM) angles radiatively.

Among other, related, work we should mention that Zoupanos [13] has proposed an approach to calculate the Cabibbo angle in the context of horizontal symmetries; basically, he chooses as his eigenstates one arrangement of the $q=\frac{2}{3}$ quarks, but a 
different arrangement of the $q=-\frac{1}{3}$ quarks. We do not fully understand the implications of such an approach.

\section{The fermion sector}

Since three families have now been essentially observed we consider a horizontal $\mathrm{SU}(3)_{\mathrm{H}}$ gauge group. The full symmetry group is $\mathrm{SU}(3)_{\mathrm{H}} \times \mathrm{SU}(3)_{\mathrm{c}} \times \mathrm{SU}(2) \times \mathrm{U}(1)$. The three families transform according to the fundamental 3 of $\mathrm{SU}(3)_{\mathrm{H}}$. The fermion content is:

$\begin{array}{llllll}\longleftrightarrow & \longleftarrow & \longleftrightarrow \\ \left(\begin{array}{l}\nu_{1} \\ \mathrm{e}\end{array}\right)_{\mathrm{L}} & \left(\begin{array}{l}\nu_{2} \\ \mu\end{array}\right)_{\mathrm{L}} & \left(\begin{array}{c}\nu_{3} \\ \tau\end{array}\right)_{\mathrm{L}} & \nu_{1 \mathrm{R}} & \nu_{2 \mathrm{R}} & \nu_{3 \mathrm{R}} \\ \left(\begin{array}{l}\mathrm{u} \\ \mathrm{d}\end{array}\right)_{\mathrm{L}} & \left(\begin{array}{l}\mathrm{c} \\ \mathrm{s}\end{array}\right)_{\mathrm{L}} & \left(\begin{array}{l}\mathrm{t} \\ \mathrm{b}\end{array}\right)_{\mathrm{L}} & \mathrm{u}_{\mathrm{R}} & \mathrm{c}_{\mathrm{R}} & \tau_{\mathrm{R}} \\ \mathrm{t}_{\mathrm{R}}\end{array}$

No change has occurred in the vertical sector. Several comments are necessary:

(a) If a fourth family is present, the horizontal gauge group can be expanded to SU(4), the families transforming as a fundamental rep of SU(4). Because of the high mass of the fourth generation, we might expect that an approximate $\mathrm{SU}(3)_{\mathbf{H}}$ would hold for the lightest three families. We will discuss this briefly at the end.

(b) To avoid anomalies, a right-handed neutrino triplet is necessary. Introducing right-handed Weyl fields for the neutrinos makes the $\mathrm{SU}(3)_{\mathrm{H}}$ vector like and therefore anomaly free.

(c) Because of the $\mathrm{SU}(2)_{\mathrm{L}}$ symmetry both components of the doublets must transform under the same way under $\mathrm{SU}(3)_{\mathrm{H}}$. The anomaly constraints then force the transformation of the right-handed fields to be the same. The gauge fields now consist of the eight $\mathrm{SU}(3)_{c}$ gluons, a triplet $\mathrm{W}$ of $\mathrm{SU}(2)_{\mathrm{L}}$, the weak hyperchange generator $\mathrm{B}$, and an octet $\mathrm{F}$ of electrically neutral $\mathrm{SU}(3)_{\mathrm{H}}$ generators.

\section{The Higgs sector}

To break the symmetry we will add Higgs fields. We do not a priori rule out dynamical symmetry breaking, in a technicolor sense, so we only use Higgs representations which could originate from condensates of fermions transforming identically with the low energy fermions. To be definite and to avoid unnecessary complication we will take a color singlet set of Higgs fields $\phi_{\alpha}^{i}$ transforming as an octet under $\mathrm{SU}(3)_{\mathrm{H}}$, doublet under $\mathrm{SU}(2)_{\mathrm{L}}$, with hypercharge +1 . These Higgs fields will give rise to the fermion masses, and to masses for the ordinary W's and $Z$ 's. To give masses to the flavor bosons $F$, some of which will be much heavier than the $\mathrm{Z}$ bosons, we have chosen a Higgs sextet $\psi_{\alpha \beta}$, which is an $\mathrm{SU}(2)_{\mathrm{L}}$ singlet. 
This sextet will also give rise to Majorana mass terms for the right-handed neutrinos. Consequently the flavor mass scale is intimately connected with neutrino masses. To obtain a realistic mass spectrum for the fermions we will also require a $\mathrm{SU}(3)_{\mathrm{H}}$ singlet $\sigma_{i}$, which is a doublet of $\mathrm{SU}(2)_{\mathrm{L}}$ and has hypercharge +1 . This is a usual Weinberg-Salam doublet Higgs boson.

We have not explicitly included a Higgs field which is an $\mathrm{SU}(3)_{\mathrm{H}}$ triplet and a singlet under $\mathrm{SU}(2)_{\mathrm{L}} \times \mathrm{U}(1)$. Although this is allowed by our philosophy, explicit calculation has revealed that the triplet gets "locked" in with the sextet and does not provide any additional information or freedom. For the sake of clarity we will neglect it here.

\section{Minimizing the Higgs potential}

Even with the restricted set of Higgs representations as outlined above, minimizing the complete Higgs potential is a formidable task. Since several physics choices are involved, we will present the arguments in considerable detail. First we will be led to a certain minimum, but it will not be satisfactory. Then with $S U(3)$ rotations we will obtain other minima. All of them either lead to no Cabibbo angle or to a disagreement with the $\mathrm{K}_{\mathrm{L}}-\mathrm{K}_{\mathrm{S}}$ mass difference.

We have the fields $\sigma^{i}, \phi_{\beta}^{i \alpha}, \psi_{\alpha \beta}$ where the roman (greek) indices represent the $\mathrm{SU}(2)_{L}\left(\mathrm{SU}(3)_{\mathrm{H}}\right)$ indices. The complete potential is:

$$
\begin{aligned}
V= & V(\sigma, \phi)+V(\psi, \sigma, \phi) \\
V(\dot{\sigma}, \phi)= & -\mu_{1}^{2} \sigma^{2}+h_{1} \sigma^{4}-\mu_{2}^{2} \operatorname{Tr} \phi^{i} \phi_{i}+g_{1}\left(\operatorname{Tr} \phi^{i} \phi_{i}\right)^{2} \\
& +g_{2} \operatorname{Tr} \phi^{i} \phi_{j} \operatorname{Tr} \phi_{i} \phi^{i}+g_{3} \operatorname{Tr} \phi^{i} \phi^{i} \operatorname{Tr} \phi_{i} \phi_{i}+g_{4} \operatorname{Tr} \phi^{i} \phi_{j} \phi^{i} \phi_{j} \\
& +g_{5} \operatorname{Tr} \phi^{i} \phi_{j} \phi_{i} \phi^{i}+g_{6} \operatorname{Tr} \phi^{i} \phi_{i} \phi_{j} \phi^{i}+g_{7} \operatorname{Tr} \phi^{i} \phi_{j} \phi^{i} \phi_{i} \\
& +f_{1} \sigma^{i} \sigma_{i} \operatorname{Tr} \phi^{i} \phi_{i}+f_{2} \sigma^{i} \sigma_{j} \operatorname{Tr} \phi_{i} \phi^{i}, \\
V(\psi, \sigma, \phi)= & -m^{2} \operatorname{Tr} \psi^{+} \psi+\frac{1}{2} \lambda_{1}\left(\operatorname{Tr} \psi^{+} \psi\right)^{2}+\frac{1}{2} \lambda_{2} \operatorname{Tr} \psi^{+} \psi \psi^{+} \psi \\
& +\beta_{1} \operatorname{Tr} \psi^{+} \psi \operatorname{Tr} \phi^{i} \phi_{i}+\beta_{2} \sigma^{i} \sigma_{i} \operatorname{Tr} \psi^{+} \psi+\beta_{3} \phi_{i \beta}^{\alpha} \psi^{\beta \gamma} \phi_{\gamma}^{i \delta} \psi_{\delta \alpha} \\
& +\beta_{5} \operatorname{Tr} \phi_{i} \phi^{i} \psi^{+} \psi+\beta_{4} \operatorname{Tr} \phi^{i} \phi_{i} \psi^{+} \psi
\end{aligned}
$$

Note that the fermion masses come only from the SU(3) singlet and octet Higgs fields. The couplings of these fields to the leptons and quarks are:

$$
\mathscr{L}_{y 18}=\sum_{\mathrm{R}, \mathrm{L}}\left\{y_{\mathrm{LR}} \bar{L}_{\alpha i} \phi_{\beta}^{i \alpha} R^{\beta}+Z_{\mathrm{RL}} \bar{L}_{\alpha i} \sigma^{i} R^{\alpha}\right\}+c \mathrm{c}+h \nu_{\mathrm{R}}^{c \alpha} \psi^{\alpha \beta} \nu_{\mathrm{R}}^{\beta},
$$

where the sum is over all the allowed representations of quarks and leptons.

We shall choose the vacuum expectation values of the fields $\phi$ and $\sigma$ to be charge conserving, i.e. with $\left(T_{3}+\frac{1}{2} Y\right) \equiv Q, Q\langle\phi\rangle=Q\langle\sigma\rangle=0$. Furthermore, we shall choose $\phi$ to be a real octet. The first choice is easily justified since we want to conserve 
electric charge. The second choice is not as easy to justify. Arbitrary large phases may introduce large $C P$ violation at the tree level which are not allowed experimentally. However, no real solid argument may be made for this choice: we impose it by fiat. We shall come back to this point later.

Having chosen the octet real, we can now choose it to be diagonal by an appropriate $\mathrm{SU}(3)$ rotation, i.e.

$$
\phi^{i}=a_{3} \lambda_{3}+a_{8} \lambda_{8} .
$$

Note that the fermion mass matrix is now automatically diagonal as the SU(3) symmetry restricts severely the number of allowed couplings. Consequently there will not be any CKM angles at tree level. We must generate them radiatively. We find the last point of great interest. Indeed the CKM angles must represent the breakdown of the horizontal symmetry. Their smallness indicates that they could be generated radiatively. Note also that the absence of cross-generation mixing at the tree level is a direct consequence of our assumption of reality of the octet.

Let $M_{\mathrm{u}}^{\phi}$ be the mass matrix for the up quarks $(\mathrm{u}, \mathrm{c}, \mathrm{t})$ generated from the octet. Clearly $M_{\mathrm{d}}^{\phi}$, the same quantity for the down quark, is proportional to $M_{\mathrm{u}}^{\phi}$. However, both matrices are traceless and if the octet were the only contribution to the fermion mass matrices the relations $m_{\mathrm{t}} \approx m_{\mathrm{c}}+m_{\mathrm{u}}$ would follow! We can now understand the role of the singlet field $\sigma^{i}$. It adds a multiple of the identity to the mass matrix and breaks this relation. However it now follows that $3\langle\sigma\rangle y_{\mathrm{t}}=\operatorname{Tr}\left(M_{\mathrm{u}}^{\phi}+M_{\mathrm{u}}^{\sigma}\right)=$ $m_{\mathrm{t}}+m_{\mathrm{c}}+m_{\mathrm{u}}$. The phase of the masses has been chosen positive arbitrarily, and $y_{\mathrm{t}}$ is a Yukawa coupling constant. Since $m_{\mathrm{t}} \gg m_{\mathrm{c}} \gg m_{\mathrm{u}}$ we see that $y_{\mathrm{t}}|\langle\sigma\rangle| \sim m_{\mathrm{t}}$. Because of the large discrepancy in the mass scale of the different generations we must now choose the parameters of the octet to guarantee a near cancellation for $m_{\mathrm{u}}$ and $m_{\mathrm{c}}$. This is very unnatural. A more realistic possibility is to choose $\langle\phi\rangle \sim-\lambda_{8}$. Then by adjusting one parameter we can choose $m_{\mathrm{u}}=m_{\mathrm{c}}=0, m_{\mathrm{t}} \sim\langle\sigma\rangle$. This we will do: we arbitrarily choose $\langle\phi\rangle$ in the $\lambda_{8}$ direction and adopt the point of view that the singlet and octet get their vacuum values from similar "condensates", so that to the tree level $m_{\mathrm{u}} \approx m_{\mathrm{c}} \approx 0$ and similarly for leptons and down quarks. The light masses might also be generated by radiative corrections in this approach.

Having chosen the form of the octet and singlet vacuum values we may now solve the equations resulting from $V(\psi, \phi, \sigma)$ for the sextet vacuum values. We will again assume that the sextet minimum is real. The same objection applies here as the reality of the octet: we impose it by fiat. Using an octet and singlet of the form:

$$
\langle\phi\rangle=\left(\begin{array}{ccc}
-v & 0 & 0 \\
0 & -v & 0 \\
0 & 0 & +2 v
\end{array}\right), \quad\langle\sigma\rangle=v,
$$

we find a set of equations for $\psi_{\alpha \beta}$ of the form

$$
0=-m 2 \psi_{\alpha \beta}+\lambda_{1}\left(\operatorname{Tr} \psi^{2}\right) \psi_{\alpha \beta}+\lambda_{2} \psi_{\alpha \mu} \psi^{\mu \nu} \psi_{\nu \beta}+R_{\alpha \beta}[\psi]
$$


The matrix $R[\psi]$ is linear in $\psi$ and given by $R_{\alpha \beta}[\psi]=A_{\alpha \beta} \psi_{\alpha \beta}$, where the matrix $\mathrm{A}_{\alpha \beta}$ has the form

$$
A=\left(\begin{array}{lll}
a & a & b \\
a & a & b \\
b & b & c
\end{array}\right)
$$

where

$$
\begin{aligned}
& a=6 v^{2} \beta_{1}+\beta_{2} w^{2}+\beta_{3} v^{2}+v^{2}\left(\beta_{4}+\beta_{5}\right), \\
& b=6 v^{2} \beta_{1}+\beta_{2} w^{2}-2 v^{2} \beta_{3}+\frac{5}{2} v^{2}\left(\beta_{4}+\beta_{5}\right), \\
& c=6 v^{2} \beta_{1}+\beta_{2} w^{2}+4 v^{2} \beta_{3}+4 v^{2}\left(\beta_{4}+\beta_{5}\right) .
\end{aligned}
$$

Because of the symmetry of $A$, an obvious solution presents itself, indeed:

$$
\psi=\left(\begin{array}{lll}
\psi_{11} & \psi_{11} & \psi_{13} \\
\psi_{11} & \psi_{11} & \psi_{13} \\
\psi_{13} & \psi_{13} & \psi_{33}
\end{array}\right)
$$

leading to

$$
\begin{gathered}
\psi_{33}^{2}=\left(\frac{b-a}{2 b-a-c}\right)^{2} \frac{m^{2}}{\lambda_{1}+\lambda_{2}}\left\{1-\frac{b^{2}-a c}{m^{2}(2 b-a-c)} \frac{2 \lambda_{1}+\lambda_{2}}{\lambda_{2}} \frac{(b-a)(b-c)}{m^{2}(2 b-a-c)}\right\}, \\
4 \psi_{11}^{2}=\left(\frac{b-c}{2 b-a-c}\right)^{2} \frac{m^{2}}{\lambda_{1}+\lambda_{2}}\left\{1-\frac{b^{2}-a c}{m^{2}(2 b-a-c)}-2 \frac{\lambda_{1}+\lambda_{2}(b-c)(b-a)}{\lambda_{2} m^{2}(2 b-a-c)}\right\}, \\
\psi_{13}^{2}=\psi_{11} \psi_{33}-\frac{1}{2 \lambda_{2}} \frac{(b-a)(b-c)}{(b-a)+(b-c)} .
\end{gathered}
$$

Note that if we assume $m^{2} \gg v^{2}$ the scale of the sextet VEV is set by $m^{2} /\left(\lambda_{1}+\lambda_{2}\right)$, not by $\langle\phi\rangle$ or $\langle\sigma\rangle$. We expect that $\langle\psi\rangle$ will be much larger than $\langle\phi\rangle$ or $\langle\sigma\rangle$ guided by the fact that FCNC are not seen with any appreciable strength. Hence a good approximation to $\langle\psi\rangle$ should be given by

$$
\begin{aligned}
& \psi_{33}^{2} \cong\left(\frac{b-a}{2 b-a-c}\right)^{2} \frac{m^{2}}{\lambda_{1}+\lambda_{2}}, \\
& 4 \psi_{11}^{2} \cong\left(\frac{b-c}{2 b-a-c}\right)^{2} \frac{m^{2}}{\lambda_{1}+\lambda_{2}}, \\
& \psi_{13}^{2} \cong \psi_{11} \psi_{33} .
\end{aligned}
$$

Now we can determine the mass matrix for the flavor bosons using the sextet:

$$
\langle\psi\rangle=\left(\begin{array}{ccc}
\eta & \eta & \mu \\
\eta & \eta & \mu \\
\mu & \mu & \rho
\end{array}\right), \quad \text { with } \mu^{2} \simeq \eta \rho .
$$


The general form for the gauge boson mass matrix yields a block diagonal matrix, with mixing between $F_{1}, F_{4}$, and $F_{8} ; F_{2}$ and $F_{7}$; and $F_{3}$ and $F_{5} . F_{6}$ is unmixed.

It is straightforward to show that both $2 \times 2$ matrices and the $3 \times 3$ matrix have a zero eigenvalue. Consequently there are three light flavor bosons, light meaning at the level of a $\mathrm{Z}$-mass (set by the scale of the octet vacuum expectation value).

The group theoretical reason for the occurrence of the three zero mass eigenstates is the fact that $\mathrm{SU}(3)$ was broken down to $\mathrm{SU}(2)$ by the sextet. The octet further breaks the $\mathrm{SU}(2)$. This mechanism is exciting since one "naturally" has two mass scales for the flavor bosons. However, the diagonalization of the mass matrix reveals a mixing of the $F_{1}-F_{2}$ flavor bosons which could potentially lead to a large contribution to the $K_{L}-K_{S}$ mass difference from the light flavor bosons. As we will see in a moment, this contribution can be rotated away. In fact, we can use $\mathrm{SU}(3)$ rotations to obtain a new form for the minimum which naturally suppresses the $\mathrm{K}_{\mathrm{L}}-\mathrm{K}_{\mathrm{S}}$ problem.

Indeed any transformation which commutes with $\lambda_{8}$ leaves the octet VEV invariant. A clue to a possible satisfactory minimum is evident from the form of the above mass matrix. Note that the two $2 \times 2$ blocks are identical in structure. One of the blocks contains $F_{2}$ which mediates $s-d$ transitions, while the other block contains an $\mathrm{F}_{3}$ which is diagonal. Suppose for a moment that the second block contained $F_{1}$ instead of $F_{3}$. There would then be a symmetry under $F_{1} \leftrightarrow F_{2}$ leading to a possible cancellation of the contributions to the $K_{L}-K_{S}$ mass difference.

Since the massless combinations are the cause of the deficiencies, we only need consider them for the moment. Since we wish to rotate $F_{1}$ into $F_{3}$ we can use a $F_{2}$ rotation. Indeed, using the $S U(3)$ rotation

$$
\left(\begin{array}{ccc}
\cos \theta & +\sin \theta & 0 \\
-\sin \theta & \cos \theta & 0 \\
0 & 0 & 1
\end{array}\right)
$$

one finds for arbitrary coefficients $A, B$,

$$
\begin{aligned}
A \lambda_{2}+B\left(\lambda_{5}-\lambda_{7}\right) \rightarrow A \lambda_{2}+B\left[(\cos \theta-\sin \theta) \lambda_{5}-(\cos \theta+\sin \theta) \lambda_{7}\right], & \\
A \lambda_{3}+B\left(\lambda_{4}-\lambda_{6}\right) \rightarrow & \left(\cos ^{2} \theta-\sin ^{2} \theta\right) A \lambda_{3}-2 \sin \theta \cos \theta \lambda_{1} A \\
& +B\left[(\cos \theta-\sin \theta) \lambda_{4}-(\cos \theta+\sin \theta) \lambda_{6}\right] .
\end{aligned}
$$

Hence, if we choose $\cos \theta= \pm \sin \theta=\sqrt{\frac{1}{2}}$, we find

$$
\left.\begin{array}{c}
A \lambda_{2}+B\left(\lambda_{5}-\lambda_{7}\right) \rightarrow A \lambda_{2}-\sqrt{\frac{1}{2}} \lambda_{7} B \\
A \lambda_{3}+B\left(\lambda_{4}-\lambda_{6}\right) \rightarrow-A \lambda_{1}-\sqrt{\frac{1}{2}} \lambda_{6} B
\end{array}\right\} \quad \cos \theta=+\sin \theta,
$$

With the above rotations we could establish a symmetry between $F_{1}$ and $F_{2}$. 
We find:

$$
\begin{aligned}
& \langle\psi\rangle \rightarrow\left(\begin{array}{ccc}
2 \eta & 0 & \mu \sqrt{2} \\
0 & 0 & 0 \\
\mu \sqrt{2} & 0 & \rho
\end{array}\right), \quad \cos \theta=+\sin \theta, \\
& \langle\psi\rangle \rightarrow\left(\begin{array}{ccc}
0 & 0 & 0 \\
0 & 2 \eta & \mu \sqrt{2} \\
0 & \mu \sqrt{2} & \rho
\end{array}\right), \quad \cos \theta=-\sin \theta .
\end{aligned}
$$

Let us concentrate on the second case for the moment. The mass matrix is again given in block diagonal form:

$F_{2}, F_{5}:$

$$
\left(\begin{array}{c:c}
4 \eta^{2}+2 \mu^{2} & \mu \sqrt{2}(2 \eta+\rho) \\
\hline \mu \sqrt{2}(2 \eta+\rho) & 2 \mu^{2}+\rho^{2}+\delta^{2}
\end{array}\right)
$$

$F_{1}, F_{4}:$

$$
\left(\begin{array}{c:c}
4 \eta^{2}+2 \mu^{2} & \mu \sqrt{2}(2 \eta+\rho) \\
\hline \mu \sqrt{2}(2 \eta+\rho) & 2 \mu^{2}+\rho^{2}+\delta^{2}
\end{array}\right)
$$

$F_{7}$ : No mixing. $\delta^{2}+4 \eta^{2}+8 \mu^{2}-4 \eta \rho+\rho^{2}$;

$$
F_{3}, F_{8}, F_{6} \text { : }
$$

$$
\begin{array}{|c|c|c}
3 & 6 & 8 \\
8 \eta^{2}+2 \mu^{2} & -\mu \sqrt{2}(6 \eta+\rho) & \frac{2 \mu^{2}-8 \eta^{2}}{\sqrt{3}} \\
\hline & \delta^{2}+4 \eta^{2}+8 \mu^{2}+\rho^{2}+4 \eta \rho & \frac{\mu \sqrt{2}(2 \eta-\rho)}{\sqrt{3}} \\
\hline & & \frac{1}{3}\left(8 \eta^{2}+8 \rho^{2}+2 \mu^{2}\right)
\end{array}
$$

where we have included the contribution from the octet to the mass matrix $\left(\delta^{2}\right)$. Again we find in the limit $\delta^{2}=0, \mu^{2}=\eta \rho$ that there are three zero eigenvalues, hence three light bosons. Note that the mass matrix is invariant under the following substitutions:

$$
F_{1} \leftrightarrow F_{2}, \quad F_{4} \leftrightarrow F_{5}
$$

This symmetry guarantees no contribution to the $\mathrm{K}_{\mathrm{L}}-\mathrm{K}_{\mathrm{S}}$ mass difference. Unfortunately it also guarantees no Cabibbo mixing of the d quark with anything. 
To prove this last statement we shall show that there is a global symmetry left over which forbids the $K_{L}-K_{S}$ transition and the Cabibbo mixing. The global symmetry is the $\mathrm{U}(1)$ rotation

$$
\left(\begin{array}{ccc}
\mathrm{e}^{i \alpha} & 0 & 0 \\
0 & 1 & 0 \\
0 & 0 & 1
\end{array}\right)
$$

We must prove that the spontaneously broken theory is invariant under the above transformation. Because up to an overall phase transformation it is an SU(3) rotation, it is clear that all currents are invariant as the theory was originally invariant. We only need to concentrate on the symmetry breaking parts of the lagrangian, i.e. the mass terms, and the couplings to the Higgs bosons.

\section{The mass terms}

Let us first examine the fermion mass terms. For concreteness we only look at the lepton masses. Since the lepton Dirac mass terms are diagonal, and only e transforms, the mass terms are invariant. Since using the form of the sextet VEV, the $\nu_{\mathrm{eR}}$ does not get a Majorana mass term, the Majorana mass is invariant.

Now consider to the gauge boson mass matrices. Clearly the ordinary $\mathrm{W}$ and $\mathrm{Z}$ mass matrix is invariant. Furthermore we can test the invariance of the flavor bosons mass matrix by simply restricting ourselves to the $2 \times 2$ blocks. It is not hard to show that the $2 \times 2$ blocks (1) and (2) are invariant under the charge rotation which in the F space takes the form:

$$
\begin{aligned}
& \mathrm{F}_{1}-i \mathrm{~F}_{2} \rightarrow \mathrm{e}^{i \alpha}\left(\mathrm{F}_{1}-i \mathrm{~F}_{2}\right), \\
& \mathrm{F}_{4}-i \mathrm{~F}_{5} \rightarrow \mathrm{e}^{i \alpha}\left(\mathrm{F}_{4}-i \mathrm{~F}_{5}\right) .
\end{aligned}
$$

By hermiticity and SU(3) invariance of the original lagrangian, all other terms are also invariant. Consequently we have proved that "electron number" or more generally first generation number is conserved. Consequently

$$
\mathbf{s} \overline{\mathrm{d}} \rightarrow \overline{\mathrm{s}} \mathrm{d} \quad \text { or } \quad c \overline{\mathrm{u}} \rightarrow \overline{\mathrm{u}} \mathrm{c}
$$

transitions are forbidden as claimed. Unfortunately, this also implies that no ūc, $\overline{\mathrm{ds}}$, etc. ... couplings are allowed, hence no Cabibbo mixing can be generated.

With a little effort one can check that all rotations give the same results. One can also see that a choice of

$$
\psi=\left(\begin{array}{ccc}
0 & \psi_{12} & 0 \\
\psi_{12} & 0 & 0 \\
0 & 0 & \psi_{33}
\end{array}\right)
$$

gives the same physics, and corresponds to an alternative original minimum of ref. [8]. This minimum corresponds to $\mathrm{SU}(3) \rightarrow \mathrm{O}(3)$ while those discussed earlier 
correspond to $\mathrm{SU}(3) \rightarrow \mathrm{SU}(2)$. This exhausts all the possibilities; every one either produces a large $\mathrm{K}_{\mathrm{L}}-\mathrm{K}_{\mathrm{S}}$ mass difference and a Cabibbo angle, or a satisfactory $\mathrm{K}_{\mathrm{L}}-\mathrm{K}_{\mathrm{S}}$ mass difference and no Cabibbo angle. (Note that since the theory is flavor-symmetric, all statements about the sd vertex apply equally to the cū vertex, and to $\mathrm{D}^{0}-\overline{\mathrm{D}^{0}}$ mixing.)

It is interesting to note that radiative corrections do generate diagonal mass terms for the lighter fermions. Whether these are realistic depends on the details of the model and will be affected by the way CKM angles are generated.

Since the mechanism of having flavor bosons on two mass scales could be more general, we go now to describe some of its predictions in the next section.

\section{Flavor changing and diagonal neutral currents}

As we have said, the structure of the model suggests a number of interesting experimental consequences. These are present for symmetry reasons, and could well appear experimentally even though the absence of CKM angles in our model indicates its physics is not complete. Since experimental clues to the flavor problem are so badly needed, it is important to be sensitive to every possibility for finding them - eventually they will appear somewhere.

Since the model is not complete we will give approximate expressions for the processes of interest. To see what happens, first we note that there is a light horizontal boson in the $F_{1}-F_{4}$ sector, one in the $F_{2}-F_{5}$ sector, and one in the $F_{3}-F_{6}-F_{8}$ sector, while $F_{7}$ is heavy. If we were to carry out the full calculation we would diagonalize these, obtaining the masses and effective mixing angles for the coupling to fermions. Without completing the full diagonalization one can see that all mixings are large, and none of the F's tend to uncouple. The light masses come out to be $m_{\mathrm{Z}}$, or a few times that if $1-\mu^{2} / \eta \rho$ is somewhat different from zero. Consequently, for estimates we use $m_{\mathrm{F}} \approx 3 m_{\mathrm{Z}}$, and assume a typical mixing angle $\sin \alpha=\frac{1}{2}$ at each vertex.

In order to give estimates for processes mediated by horizontal gauge bosons we need to know the horizontal gauge coupling $g_{\mathrm{H}}$. In the present model $g_{\mathrm{H}}$ is a free parameter, but it becomes calculable under the following assumption.

We suppose that the model can be unified within some grand unified group $G$ at some high energy, and further that the extra particles required to form complete representations of $\mathrm{G}$ have masses characteristic of the scale, $M_{\mathrm{u}}$, at which $\mathrm{G}$ breaks to $\mathrm{SU}(3) \times \mathrm{SU}(2) \times \mathrm{U}(1) \times \mathrm{SU}(3)_{\mathrm{H}}$. Then according to the standard calculations [15], if we input $\alpha$ and $\alpha_{3}$ at $M_{\mathrm{W}}$ (say) we can calculate $\sin ^{2} \theta, M_{\mathrm{u}}$ and $\alpha_{\mathrm{H}}=g_{\mathrm{H}}^{2} / 4 \pi$.

In the one-loop approximation we find

$$
\begin{aligned}
\sin ^{2} \theta_{\mathrm{W}} & =0.236, \\
M_{\mathrm{u}} & =2.8 \times 10^{13}, \\
\alpha_{\mathrm{H}} & =0.04 \approx \alpha_{2} .
\end{aligned}
$$


The calculation of $\sin ^{2} \theta_{\mathrm{W}}$ and $M_{\mathrm{u}}$ corresponds to that for the standard model with $H=9$ Higgs doublets. As a result $\sin ^{2} \theta_{\mathrm{w}}$ comes out somewhat high and $M_{\mathrm{u}}$ somewhat low. (They are both monotonic functions of $H$ in this approximation.) The latter point is not too disturbing since, as emphasized, for example, in ref. [16], if we relax somewhat the assumption that all heavy particles have masses very close to $M_{\mathrm{u}}$ it is possible to induce considerable variations in the value obtained for $M_{\mathrm{u}}$. The result for $\sin ^{2} \theta_{\mathrm{W}}$ is more disturbing and may require the abandonment of the simple one-stage unification idea described above. In any case, we may use the value of $\alpha_{\mathrm{H}}$ given above to suggest orders of magnitude for some interesting processes.

Then a typical "large" process such as

$$
\tau \rightarrow \mu \mathrm{e}^{+} \mathrm{e}^{-}
$$

will have a rate, relative to a semileptonic mode, of

$$
\Gamma\left(\tau \rightarrow \mu \mathrm{e}^{+} \mathrm{e}^{-}\right) / \Gamma(\tau \rightarrow \mu \nu \bar{\nu}) \simeq \frac{1}{2}\left[\frac{g_{\mathrm{H}}^{2}}{g_{2}^{2}} \frac{m_{\mathrm{Z}}^{2}}{m_{\mathrm{F}}^{2}} \sin ^{2} \alpha\right]^{2} \simeq 1 / 2000 .
$$

Then the branching ratio is

$$
\operatorname{BR}\left(\tau \rightarrow \mu \mathrm{e}^{+} \mathrm{e}^{-}\right) \simeq 0.17 / 2000 \approx 10^{-4} .
$$

Without a better model, we cannot predict if a given process should be at this level or a few times it or $10^{-1}$ times it, but it is clear that some processes mediated by horizontal bosons should occur at these levels. Experimentally [11], rare $\tau$ decays have been excluded at a branching ratio of about $5 \times 10^{-4}$, with less stringent limits for $b$ decay [12].

To see which processes occur, we write the fermion-gauge boson couplings. Using the charged leptons as an example (and $\mathrm{e} \rightarrow \mathrm{d}$ or $\mathrm{u} ; \mu \rightarrow \mathrm{s}$ or $\mathrm{c} ; \tau \rightarrow \mathrm{b}$ or $\mathrm{t}$ for the quarks), the interaction is $(\overline{\mathrm{L}}=(\overline{\mathrm{e}} \bar{\mu} \bar{\tau}))$

$$
\begin{aligned}
g_{\mathrm{H}} \bar{L} F^{\mathrm{A}}\left(\frac{1}{2} \lambda^{\mathrm{A}}\right) L= & \left(\frac{1}{2} g_{\mathrm{H}}\right)\left[\bar{e}\left(F_{3}+\sqrt{\frac{1}{3}} F_{8}\right) e+\bar{\mu}\left(-F_{3}+\sqrt{\frac{1}{3}} F_{8}\right) \mu\right. \\
& \left.+\bar{\tau}\left(-2 \sqrt{\frac{1}{3}} F_{8}\right) \tau\right]+\left[\bar{e}\left(F_{1}-i F_{2}\right) \mu+\bar{e}\left(F_{4}-i F_{5}\right) \tau\right. \\
& \left.+\bar{\mu}\left(F_{6}-i F_{7}\right) \tau+\text { H.C. }\right] .
\end{aligned}
$$

The allowed vertices are shown in fig. 1.
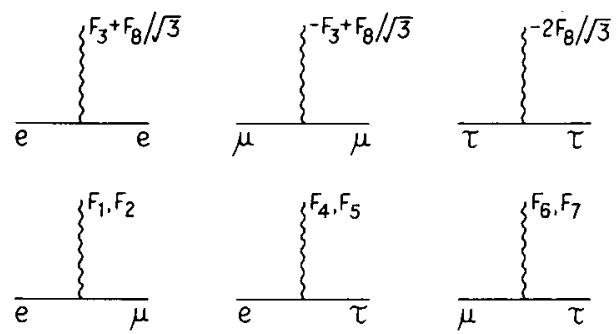

Fig. 1. The fermion-gauge boson vertices which are present in the model. $F_{1}$ and $F_{4}$ can mix, $F_{2}$ and $F_{5}$ can mix, and $F_{3}, F_{6}, F_{8}$ can mix. With this information one can construct the allowed interactions. 

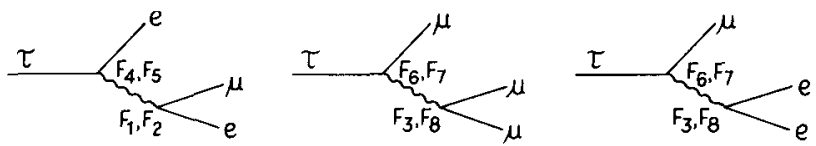

(a)

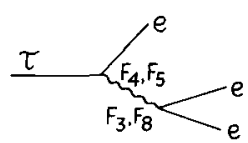

(b)

Fig. 2. Decays shown in (a) are allowed, while (b) is forbidden.

These interactions lead to a series of interesting decays and rates.

(i) Note that the electron and muon neutral currents will not be equal; that is, there is a violation of $\mathrm{e} / \mu$ (and $\tau$ ) universality. The relative amplitude could be of order

$$
\frac{\sin ^{2} \alpha g_{\mathrm{H}}^{2} / m_{\mathrm{F}}^{2}}{g_{2}^{2} / m_{\mathrm{Z}}^{2}} \simeq 1 / 36,
$$

so, with interference, differences up to $10 \%$ in normalization for $\mathrm{eN} \rightarrow \mathrm{eX}$ and $\mu \mathrm{N} \rightarrow \mu \mathrm{X}$, or of $\mathrm{e}^{+} \mathrm{e}^{-} \rightarrow \mathrm{e}^{+} \mathrm{e}^{-}$and $\mathrm{e}^{+} \mathrm{e}^{-} \rightarrow \mu^{+} \mu^{-}$and $\mathrm{e}^{+} \mathrm{e}^{-} \rightarrow \tau^{+} \tau^{-}$, could occur. Similar factorization violations would occur in relating neutrino and charged lepton processes. Without further information it is not possible to say which would be larger.

(ii) The decays of fig. $2 \mathrm{a}$ for the $\tau$ are allowed. For example, $\mathrm{F}_{1}$ and $\mathrm{F}_{4}$ mix so the first is permitted and $F_{6}$ and $F_{3}$ mix so the next two can occur. But the process of fig. $2 b$ is forbidden since $F_{4}$ and $F_{5}$ do not mix with $F_{3}, F_{8}$. The three decays of fig. 2a will occur with rates of the same order of magnitude, but not equal at the factor-of-two level since the detailed gauge boson mixings depend on the horizontal mass scales. Note that this pattern is not what might be expected for FCNC from Higgs or technicolor physics, where the coupling is proportional to the mass and the process $\tau \rightarrow \mu(\mathrm{e})$ would be suppressed.
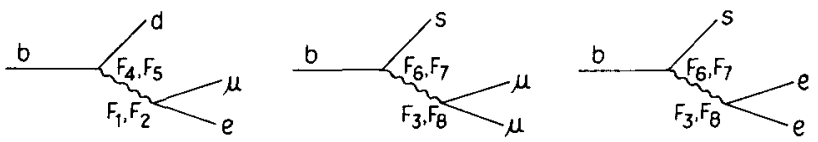

(a)

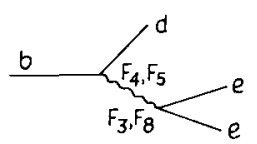

(b)

Fig. 3. Decays shown in (a) are allowed, while (b) is forbidden. 
Similar remarks apply for $b$ and $t$ decays. In particular, as shown in fig. 3, note that ( $3 \mathrm{a}$ are allowed, $3 \mathrm{~b}$ is forbidden)

$$
\mathrm{b} \rightarrow \mathrm{s} \mu \mu \quad \text { or } \quad \mathrm{b} \rightarrow \text { see }
$$

should occur with comparable rates, but not

$$
\mathrm{b} \rightarrow \mathrm{d} \mu \mu \text { or } \mathrm{b} \nrightarrow \mathrm{dee} ;
$$

that is, if $b \rightarrow X e^{+} e^{-}$is due to FCNC it must occur with a kaon in $X$. Similarly,

but

$$
\mathrm{b} \rightarrow \mathrm{d} \mu \mathrm{e}
$$

$$
\mathrm{b} \rightarrow \mathrm{s} \mu \mathrm{e}
$$

so if $b \rightarrow X \mu e, X$ must not contain an odd number of strange quarks. While vacuum $\bar{s}$ pairs may complicate checking these predictions somewhat, the situation should in principle be fairly clean.

(iii) Additional neutral current contributions will occur in decays such as $\psi \rightarrow \mathrm{f} \overline{\mathrm{f}}$, $T \rightarrow f \bar{f}, Z^{0} \rightarrow f \bar{f}$ where $f$ is any quark or lepton.

(iv) In $\mathrm{e}^{+} \mathrm{e}^{-}$processes one can have directly the production of $\tau \bar{\mu}, \mathrm{b} \overline{\mathbf{s}}$, t $\overline{\mathbf{c}}$ as in

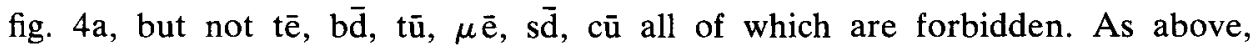
these could have amplitudes of order $5-10 \%$ of a typical $\mathrm{Z}^{0}$ contribution to $\mathrm{e}^{+} \mathrm{e}^{-}$ at a given energy (off resonance). This is just a little small to be observed at $\sqrt{s} \simeq 30 \mathrm{GeV}$ with present luminosities, but could be achieved if the luminosity increases or at higher energies. These would give very clear signals with different size jets ${ }^{\star}$.

Finally, in general there will also be flavor changing neutral currents due to horizontal Higgs. Presumably they will give effects proportional to masses, and with systematics different from flavor interactions generated by gauge bosons. In the heavier fermion interactions such effects could be of order $\left(m_{\mathrm{f}} / m_{\mathrm{h}}\right)^{2}$.

In this work we only considered the horizontal group SU(3), with the families in the fundamental representations of the horizontal gauge group. However, we have briefly considered the extension to other groups. Indeed if the horizontal group is chosen to be SU(4), there is a sequential breaking first to $\mathrm{SU}(3)$ or $\mathrm{SO}(4)$. In the $\mathrm{SU}(3)$ case any further breaking leads one to the case we have studied. The $\mathrm{SO}(4)$ case reproduces the model of Ong [4], which fails phenomenologically. Going to

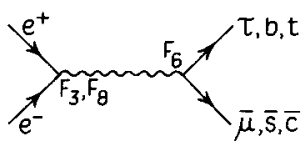

Fig. 4. The production of flavor-asymmetric jets.

* D. Meyer has emphasized that such a mechanism might give a way to find a $t$-quark with mass nearly as large as the available energy at an $\mathrm{e}^{+} \mathrm{e}^{-}$collider. 
$\mathrm{SU}(5)$ the situation is different if $\mathrm{SU}(5) \rightarrow \mathrm{SO}(5)$ in the primary breaking. We have not investigated this case.

Of course many extensions of the present framework are possible. We did not for instance try to vary the representations of the basic families. We can only say that the simplest suggestive choice fails.

We conjecture that this failure will subsist for higher groups. The CKM angles do not seem to be generated radiatively and the value of horizontal symmetries is questionable.

This research was supported in part by the U.S.D.O.E. We appreciate discussions with D. Meyer. We thank M. Yoshimura, L. Wolfenstein and S. Pakvasa for bringing the work of Yanagida and collaborators to our attention.

\section{Appendix}

We give here some useful formalism. The gauge boson octet is

$$
F=\left(\begin{array}{ccc}
F_{3}+\sqrt{\frac{1}{3}} F_{8} & F_{1}-i F_{2} & F_{4}-i F_{5} \\
F_{1}+i F_{2} & -F_{3}+\sqrt{\frac{1}{3}} F_{8} & F_{6}-i F_{7} \\
F_{4}+i F_{5} & F_{6}+i F_{7} & -2 \sqrt{\frac{1}{3}} F_{8}
\end{array}\right) .
$$

For a sextet Higgs the mass matrix gets a contribution

while for an octet Higgs it is

$$
\operatorname{Tr}\left[F^{2} \psi^{2}+F \psi F^{*} \psi\right],
$$

$$
\operatorname{Tr}\left[F^{2} \phi^{2}-F \phi F \phi\right] .
$$

The covariant derivations are

$$
\begin{aligned}
& D \mu \phi_{A}^{i}=\partial \mu \phi_{A}^{i}+\frac{1}{2} i g(\tau \cdot W \mu)^{i j} \phi_{A}^{i}+\frac{1}{2} i g^{\prime} Y B_{\mu} \phi_{A}^{i}+\frac{1}{2} i g_{\mathrm{H}} f_{A B C} F_{B \mu} \phi_{C}^{i}, \\
& D_{\mu} \psi_{a b}=\partial_{\mu} \psi_{a b}-\frac{1}{2} i g_{\mathrm{H}} F_{a c} \psi_{c b} .
\end{aligned}
$$

It is convenient to write $\psi$ as a $3 \times 3$ matrix with labels $a, b$, and $\phi$ and $F$ interchangeable as matrices or vectors, with

$$
\phi_{a b}=\sum_{A} \phi_{A} \lambda_{a b}^{A}, \quad A=1,2, \ldots 8 .
$$

The superscript $i$ is an $\mathrm{SU}(2)_{\mathrm{L}}$ index for the octet, which is simultaneously an $\mathrm{SU}(2)_{\mathrm{L}}$ doublet and a horizontal octet.

\section{References}

[1] H. Georgi, Nucl. Phys. B165 (1979) 126

[2] M. Gell-Mann, P. Ramond and R. Slansky, in Supergravity, ed. P. van Nieuwenhuizen and D.Z. Freedman (North-Holland, 1979) p. 315 
[3] E. Derman and D.R.T. Jones, Phys. Lett. 70B (1977) 449;

E. Derman, Phys. Rev. D19 (1979) 317

[4] F. Wilczek and A. Zee, Phys. Rev. Lett. 42 (1979) 421;

S. Barr and A. Zee, Phys. Rev. D17 (1978) 1854;

A. Davidson, M. Koca and K.C. Wali, Phys. Rev. Lett. 43 (1979) 92;

S. Meshkov and S.P. Rosen, Phys. Rev. Lett. 29 (1972) 19764;

C.L. Ong, Phys. Rev. D19 (1979) 2738;

R. Mohapatra, Phys. Rev. D9 (1974) 3461

[5] O.W. Greenberg, Phys. Rev. Lett. 35 (1976) 1120;

J.C. Pati, A. Salam and J. Strathdee, Phys. Lett. 58B (1975) 265;

H. Harari, Phys. Lett. 86B (1979) 83;

M.A. Shoupe, Phys. Lett. 86B (1979) 87;

G.L. Shaw and R. Slansky, Phys. Rev. D22 (1980) 1760;

M. Veltman, Pr. 2nd Workshop on Grand unification, ed. J. Leveille, L. Sulak, D. Unger (April, 1981)

[6] T. Machara and T. Yanagida, Prog. Theor. Phys. 60 (1978) 822; 61 (1979) 1434;

T. Yanagida, Phys. Rev. D20 (1979) 2986; Prog. Theor. Phys. 64 (1980) 1103

[7] Y. Chikashige, G. Gelmini, R.D. Peccei and M. Roncadelli, Phys. Lett. 94B (1980) 499

[8] L.F. Li, Phys. Rev. D9 (1974) 1723

[9] G.L. Kane and R. Thun, Phys. Lett. 94B (1980) 513

[10] R.N. Cahn and H. Harari, Nucl. Phys. B176 (1980) 135

[11] J. Dorfan, private communication;

K.G. Hayes and M.L. Perl, SLAC-PUB-2699 (Feb., 1981)

[12] K. Chadwick, Phys. Rev. Lett. 46 (1981) 88

[13] G. Zoupanos, The Cabibbo angle and the mass of the t-quark in an $S U(2)_{L} \times U(1)$ model with horizontal symmetry, Dortmund and Max-Planck Institute preprint

[14] P. Herczeg, Los Alamos preprint (June, 1981)

[15] H. Georgi, H. Quinn, and S. Weinberg, Phys. Rev. Lett. 33 (1974) 451

[16] K.T. Mahanthappa and M.A. Sher, in Unification of the fundamental particle interactions, ed. S. Ferrara, J. Ellis and P. van Nieuwenhuizen 